\title{
Investigation on Dynamic Interaction of Multiple Clearance Joints for Flap Actuation System
}

\author{
Qi Wan, Geng Liu*, Shangjun Ma and Ruiting Tong \\ Shaanxi Engineering Laboratory for Transmissions and Controls, Northwestern Polytechnical University, Xi'an 710072, China
}

\begin{abstract}
A dynamic simulation model of the flap actuation system is developed in this paper to analyze the dynamic interaction behaviours of multiple clearance joints. The nonlinear contact force model and modified Coulomb friction model are adopted in the four clearance joints to capture their motion modes, including free flight mode, impact mode and continuous mode. The results show that there exists a strong dynamic interaction between different clearance joints and one impact motion mode in a joint will immediately affect other joints. And when the system reaches a stable state, the four clearance joints almost appears the similar tendency due to the rigid connection. Therefore, in order to accurately predict the dynamic responses of multibody system, it is essential for all joints to be modelled as imperfect ones.
\end{abstract}

\section{Introduction}

As flap actuation system is widely employed in the field of aircrafts, the demands of predicting the system's dynamic characteristics accurately are becoming increasingly essential. The flap actuation system mainly consists of an actuator, linkage mechanism, flight control surface and control system, which is a high precise position servo system [1]. The actuator receives the control command of flight attitude of aircrafts, and then produces force to drive linkage mechanism connected with control surface. At last, the aim of flight direction and attitude of aircrafts is accomplished. The output angle of control surface is the main control target of the flap actuation system, and its follow-up of the command signal directly determines the flight quality of aircrafts. However, as the typical multibody mechanism with clearances, the linkage mechanism of flap actuation system has great influence on dynamic behaviors of aircrafts. Therefore, it is necessary to analyze the influence of joint clearance in the linkage mechanism on the dynamic responses of flap actuation system.

Due to the manufacturing, assembly processes, and wear errors, joint clearance is inevitable and always leads to extraordinary negative effect on the dynamic characteristic of multibody system. A number of researchers pointed that there is a strong dynamic interaction between different clearance joints and all joints should be considered as non-ideal to accurately predict the dynamic performance of aircrafts and spacecrafts. Li [2] established the dynamic model of the spacecraft with large deployable solar arrays considering multiple clearance joints and studied the effect of joint clearance on the satellite attitude under deployment disturbance. The results showed that system response with two clearance joints is not a simple superposition of that in a mechanism with one clearance joint. Bai [3] studied the dynamic responses of satellite antenna dualaxis driving mechanism with two planar revolute clearance joints and developed an optimization method to reduce the undesirable vibrations due to impact forces in clearance joints. Li [4] investigated the effects of the number of clearance joints on the dynamic characteristics of planar deployable structure based on scissor-like element, and the results indicated that the motion in one revolute clearance joint will affect the motion in the other clearance joint. However, the combination motion modes between multi-clearance joints and some inner rules existing those modes are not revealed. Muvengei [5] and Ma [6] studied the interaction and combination motion modes between clearance joints, but the slider-crank mechanism only including four simple parts is always studied and there has been less investigated on the combination motion modes and inner rules of aircrafts with imperfect revolute joints.

Therefore, in order to achieve the relationship between system responses and combination motion modes as well as dynamic interaction of clearance joints, the dynamic characteristics of flap actuation system with multiple clearance joints are investigated in this paper, which is significant for the dynamic simulation and analysis of transmission system in aerospace field.

\section{Modelling of flap actuation system with joint clearance}

\subsection{Model of revolute joint with clearance}

The perfect revolute joint assumes that the centers of journal and bearing are coincident all the time with only

Corresponding author: npuliug@nwpu.edu.cn 
relative rotation allowed, while the imperfect one means that there is a clearance between the centers of journal and bearing.

The clearance can be expressed by

$$
c=r_{B}-r_{J}
$$

where $c$ denotes the radial clearance and $r_{B}$ and $r_{J}$ are the radii of bearing and journal, respectively.

The mathematical model of the revolute clearance joint is shown in Fig. 1. XOY donates the generalized coordinate system. $O_{B}$ and $O_{J}$ are the centers of bearing and journal, respectively.

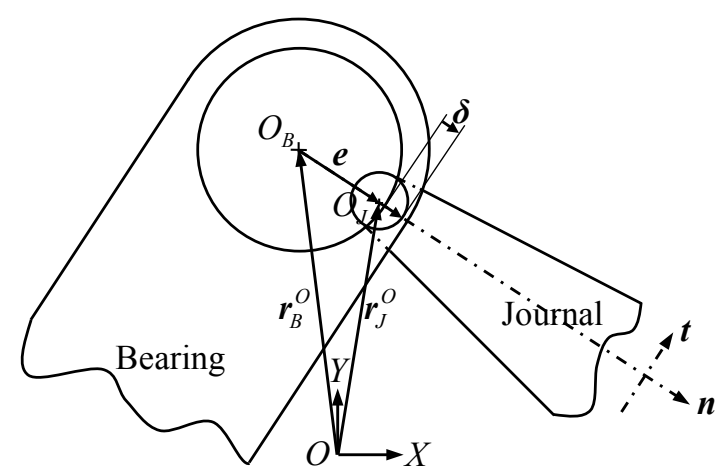

Fig. 1. Mathematical model of revolute clearance joint.

As shown in Fig. 1, the eccentricity vector which connects the centers of bearing and journal $\left(O_{B}\right.$ and $\left.O_{J}\right)$ can be written as [3-4]:

$$
\boldsymbol{e}=\boldsymbol{r}_{J}^{O}-\boldsymbol{r}_{B}^{O}
$$

where $\boldsymbol{r}_{B}^{O}$ and $\boldsymbol{r}_{J}^{O}$ are the positional vectors of centers of bearing and journal in generalized coordinate system (XOY), respectively.

The magnitude of the vector $\boldsymbol{e}$ can be described as

$$
e=\sqrt{\boldsymbol{e}^{\mathrm{T}} \boldsymbol{e}}
$$

And the normal unit vector $\boldsymbol{n}$ at collision point can be expressed as

$$
\boldsymbol{n}=\boldsymbol{e} / e
$$

The tangential unit vector at the collision point can be obtained by reversing $\mathrm{n}$ for $90^{\circ}$.

When the journal and the bearing come into contact with each other, the relative penetration depth can be calculated with respect to $e$ and $c$

$$
\delta=e-c
$$

The motion of a journal in a bearing can be divided into three categories: the free flight mode, the contact mode and the impact mode. The relative motion relationship between the journal and the bearing can be accurately determined by the relative penetration depth $\delta$. There are several situations as follows: (a) If $\delta<0$, there is no collision between two bodies and the journal moves freely within the bearing's boundaries, which means the system is in the free flight mode and the contact force between journal and bearing is equalled to zero; (b) If $\delta>0$, the journal and bearing contact with each other and the system is in the continuous contact mode; (c) If $\delta$ changes from the negative to the positive, it means the two bodies are gradually approaching. Particularly, when the relative penetration depth is equalled to zero, there is an impact force between the journal and bearing and the system is in the impact mode at that moment; (d) If $\delta$ changes from the positive to the negative, it indicates the two are gradually separating.

\subsection{Contact force model}

The "Impact-function" in multi-body dynamic software, ADAMS [7-8], which is a nonlinear spring-damper model considering the effect of energy dissipation process and is widely employed by many researchers. Ma compared the simulation results of a slider-crank mechanism with clearance joints, which the normal contact forces are respectively calculated by "Impactfunction" and the presented model, and the simulation results of those two models have a good agreement with each other [6]. Khemili studied the dynamic behavior of a planar slider-crank mechanism with clearance by "Impact-function" and verified the effectiveness of contact force model by some experiments [9]. Chen analyzed the dynamic responses of a closed high speed and heavy load press and the "Impact-function" is employed to describe the contact response between the journal and bearing. As the numerical results agree well with the experimental data, it indicates that the "Impactfunction" can accurately describe the dynamic performance of the multibody system [10]. Therefore, the "Impact-function" is used to predict the dynamic characteristics of contact-impact process for the flap actuation system, and its equations can be given by [11]

$$
F_{n}=\left\{\begin{array}{cc}
K \delta^{1.5} & \delta \leq 0 \\
K \delta^{1.5}+c_{\max }\left(\frac{\delta}{\delta_{\max }}\right)^{2}\left(3-2 \frac{\delta}{\delta_{\max }}\right) \dot{\delta} & 0<\delta<\delta_{\max } \\
K \delta^{1.5}+c_{\max } \dot{\delta} & \delta>\delta_{\max }
\end{array}\right.
$$

where $\delta$ is the relative penetration depth; $\delta_{\max }$ is the maximal penetration depth; $c_{\max }$ is the maximal damping coefficient, which is about one percent of the contact stiffness; $K$ is the generalized contact stiffness and relate to the material property and geometries of bearing and journal, which can be expressed as

$$
K=\frac{4}{3\left(\frac{1-v_{B}}{E_{B}}+\frac{1-v_{J}}{E_{J}}\right)} \sqrt{\frac{R_{B} R_{J}}{R_{B}-R_{J}}}
$$

where $R_{B}$ and $R_{J}$ are the radii of bearing and journal, respectively; and the material parameters of bearing and journal are associated with Poisson's ratio, $v_{B}, v_{J}$, and Young's modulus, $E_{B}, E_{J}$, respectively. 
The frictional characteristics of the revolute clearance joint is depicted by a modified Coulomb friction force model, and its formula is shown as [11]

$$
F_{t}=\left\{\begin{array}{cc}
-\mu_{d} \cdot \operatorname{sign}\left(v_{t}\right) F & \left|v_{t}\right|>v_{d} \\
-\operatorname{step}\left(\left|v_{t}\right|, v_{d}, \mu_{d}, v_{s}, \mu_{s}\right) \cdot \operatorname{sign}\left(v_{t}\right) & v_{s} \leq\left|v_{t}\right| \leq v_{d} \\
\operatorname{step}\left(v_{t},-v_{s}, \mu_{s}, v_{s},-\mu_{s}\right) & \left|v_{t}\right|<v_{s}
\end{array}\right.
$$

where $v_{t}$ is the relative sliding velocity at contact point; $v_{s}$ is the critical stiction transition velocity; $v_{d}$ is the friction transition velocity; $\mu_{s}$ is the static friction coefficient; $\mu_{d}$ is the dynamic friction coefficient.

\section{Simulations and results}

\subsection{System properties}

The dynamic model of flap actuation system with four clearance joins is shown in Fig. 2. The material properties of the system are shown in Table 1 and the parameters used in the dynamic simulations are presented in Table 2. The "Impact-function" is adopted in the contact force model of clearance joints, and Gear Stiff integrator (GSTIFF) is chosen in ADAMS/Solver. During the simulation, the integration tolerance is 0.001 and the step size is $0.001 \mathrm{~s}$.

\subsection{Dynamic interaction}

This section presents the dynamic interaction phenomenon by analyzing the influences of clearance on the dynamic responses of the flap actuation system. The command of actuator's output displacement is defined as $10 \mathrm{~mm}$ with a constant frequency equal to $4 \mathrm{~Hz}$, and size of the radial clearance equals $0.1 \mathrm{~mm}$ with the bearing diameter as $15 \mathrm{~mm}$. The total simulation time is $0.5 \mathrm{~s}$.
Fig. 3 shows that the existence of joint clearance has extraordinary obvious effects on the angular velocity and angular acceleration, which is obviously shaky around the ideal curve. The constant value in angular velocity correspond to the zero angular acceleration and the impulse type of angular acceleration means the abrupt increase of angular velocity, which indicates that joint clearance will cause oscillation of the system movement and lead to the worse system stability.

Table 1. Material properties.

\begin{tabular}{|c|c|c|c|}
\hline Parts & $\begin{array}{c}\text { Young's } \\
\text { modulus } \\
(\mathrm{GPa})\end{array}$ & $\begin{array}{c}\text { Poisson's } \\
\text { ratio } \\
(/)\end{array}$ & $\begin{array}{c}\text { Mass } \\
\text { density } \\
\left(\mathrm{kg} / \mathrm{m}^{3}\right)\end{array}$ \\
\hline $\begin{array}{c}\text { Actuator } \\
\text { Rod1 } \\
\text { Bearing house 1,2,3,4 } \\
\text { Inertia plate }\end{array}$ & 209 & 0.269 & 7890 \\
\hline $\begin{array}{c}\text { Rod2 } \\
\text { Lever arm1 } \\
\text { Lever arm2 }\end{array}$ & 45 & 0.33 & 2700 \\
\hline Shaft & 211 & 0.28 & 7700 \\
\hline
\end{tabular}

Table 2. Parameters used in the dynamic simulations.

\begin{tabular}{|c|c|c|c|}
\hline Parameter & Description & Value & Unit \\
\hline$\delta_{\max }$ & Maximal penetration depth & 0.01 & $\mathrm{~mm}$ \\
\hline$\mu_{s}$ & Static friction coefficient & 0.01 & $/$ \\
\hline$\mu_{d}$ & Dynamic friction coefficient & 0.01 & $/$ \\
\hline$v_{s}$ & Stiction transition velocity & 0.1 & $\mathrm{~mm} / \mathrm{s}$ \\
\hline$v_{d}$ & Friction transition velocity & 10 & $\mathrm{~mm} / \mathrm{s}$ \\
\hline
\end{tabular}

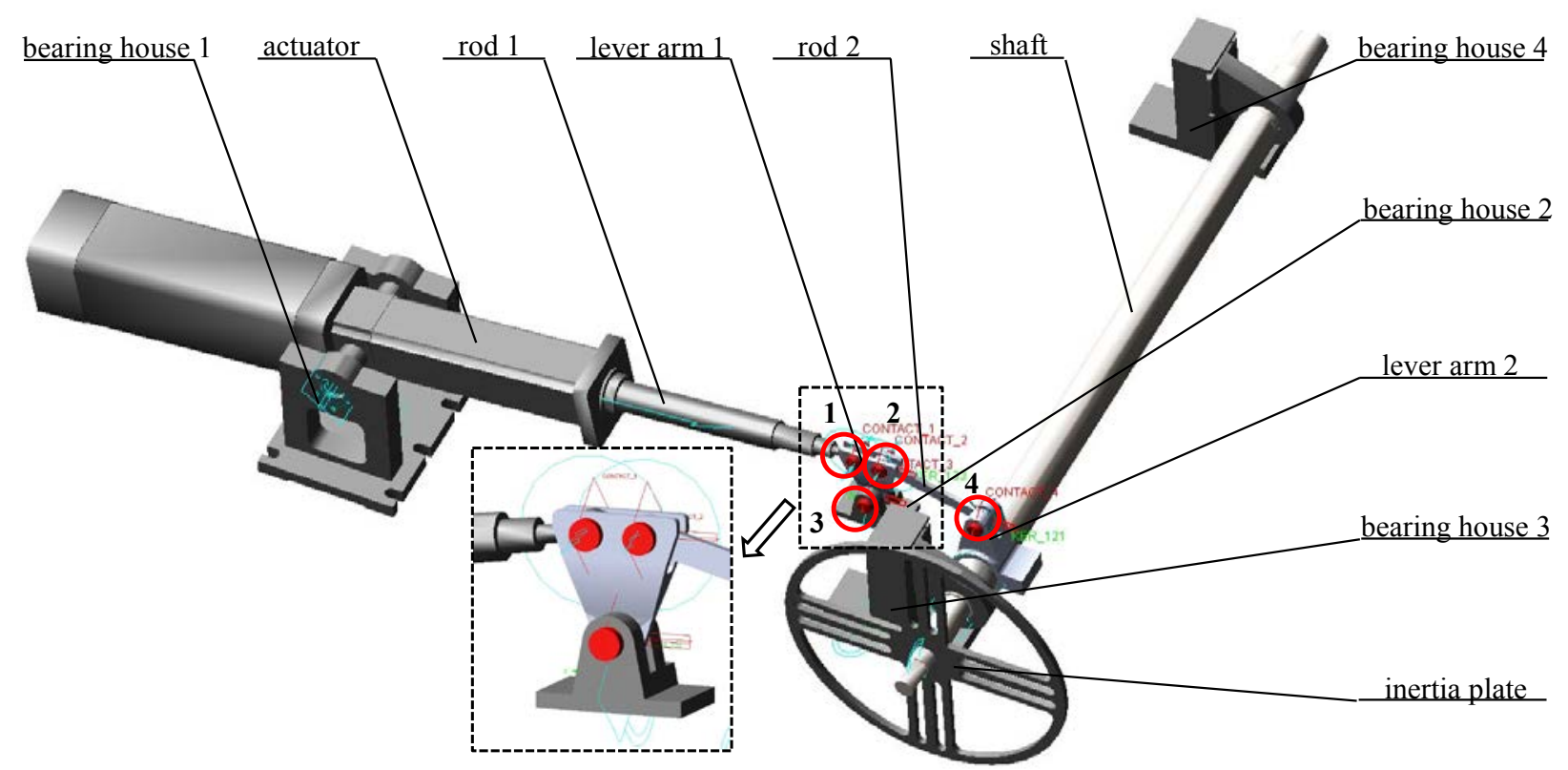

Fig. 2. Dynamic model of flap actuation system with four clearance joints. 


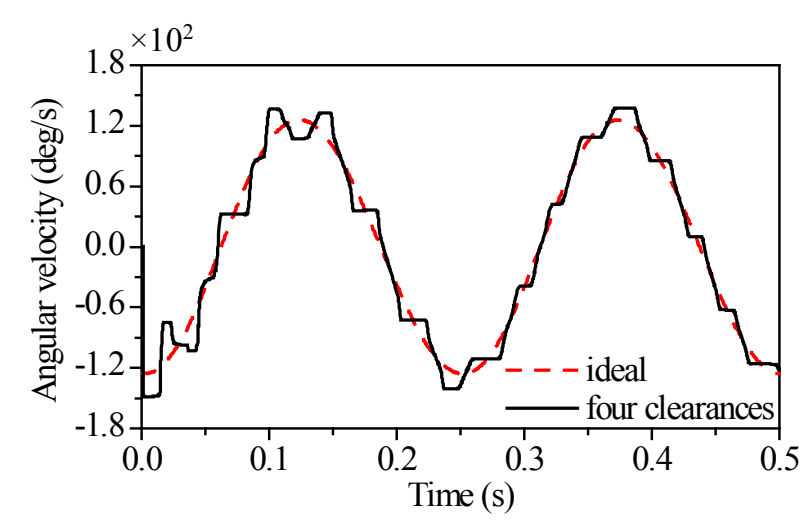

(a) Angular velocity

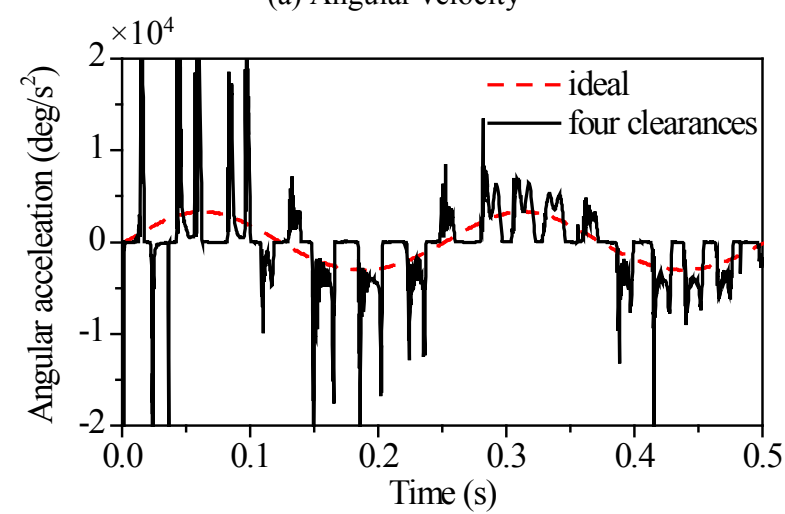

(b) Angular acceleration

Fig. 3. Dynamic responses of the system with four clearances.

Fig. 4 shows the dynamic responses of the system during the period time between 0.006 and 0.014 seconds. Contact forces at four clearance joints with five marked time indicate five combination mode, which are presented as follows.

Case (1) Impact-impact-free-free combination mode

Case (2) Free-contact-contact-contact combination mode

Case (3) Free-contact-free-contact combination mode

Case (4) Free-free-free-contact combination mode

Case (5) Contact-contact-free-impact combination mode

(1) Cases (1) (5)

Case (1) shows that there occurs an unexpected increase at the contact forces of the first and second joints, meaning that they are in impact motion, and the contact forces at the third and fourth joints are zero, indicating that the latter two joints are in free flight motion. As the fourth joint is connected with the lever arm2 and shaft of the system, the motion mode of the fourth joint can reflect the motion state of the flap actuation system. When the fourth joints is in the free flight mode, there is no reaction force acted on the shaft, and hence, the angular acceleration of the system is equalled to zero and its angular velocity keeps a constant value, as shown in the Fig. 4(b). Case (5) shows that the angular acceleration of the system changes abruptly and the angular velocity keeps increasing, since the impact force occurred in the fourth joint with the assumption that the lever arm 2 and shaft are modelled as two rigid parts.

Therefore, the change tendency of system responses can judged by the motion mode of the joint connected with the output component.

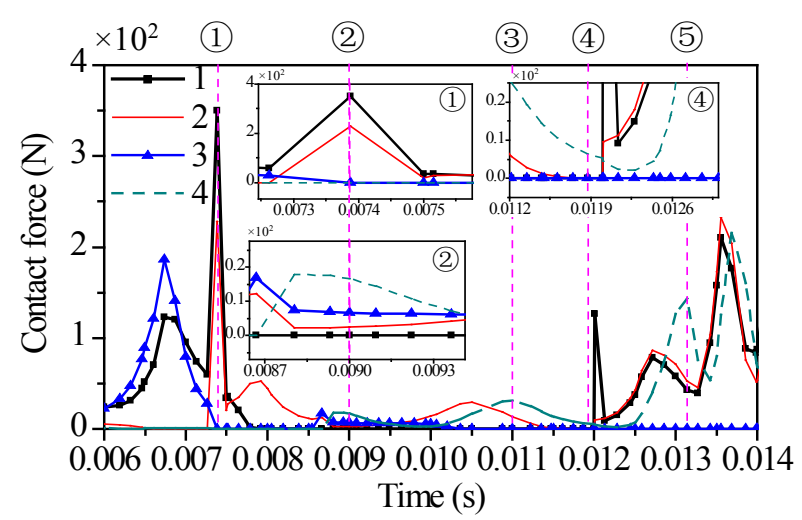

(a) Contact force

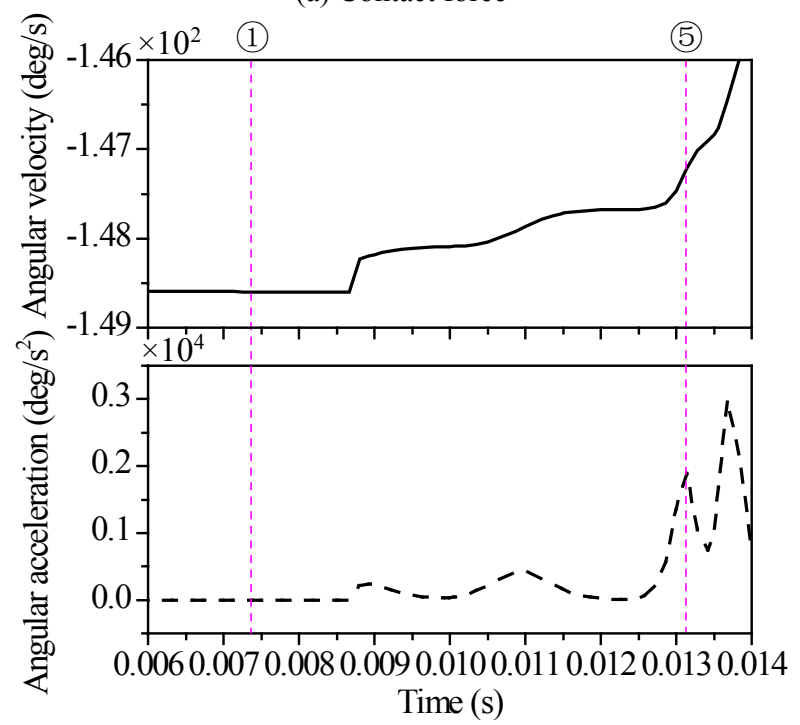

(b) Angular velocity and angular acceleration of cases (1)(5)

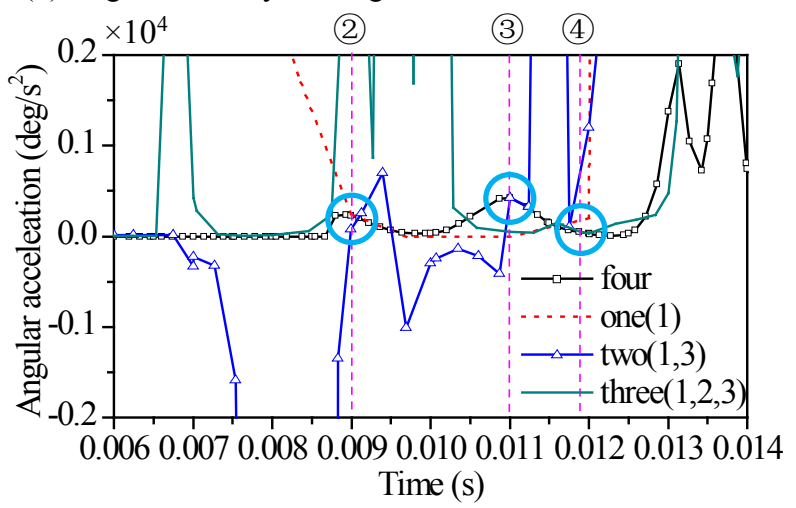

(c) Angular acceleration of cases (2)(3)(4)

Fig. 4. Dynamic responses of the system at the beginning of numerical simulation

(2) Cases (2)(3)(4)

Case (2) shows that the first joint is in the free flight mode due to the null contact force and the latter three joints are in the continuous contact mode because of the stable contact forces. It can be observed from the Fig. 4(c) that the system angular acceleration curve almost coincides with that of only the first clearance joint situation. Similar to case (2), it can be observed in case (3). When contact motion occurs in the second and fourth joints, the system angular acceleration curve almost overlap that of cases which only considers two clearance joints including the first and third joints. Similarly, this 
is evident in case (4), the system angular acceleration curve with four clearance joints case almost coincides with the one with three clearance joints, namely the first, second and third one.

Fig. 5 presents the contact forces of the four clearance joints after the system reaches a steady state, which appears that the clearance joint located closer to the input component undergoes the higher contact force and the corresponding conclusion can be obtained from the reference [6]. In addition, the second and fourth clearance joints, connected by a rigid rod, present almost the same contact force. The four clearance joints experience similar changing trends of contact force since impact mode occurred in one joint will immediately affect other joints in the rigid system.

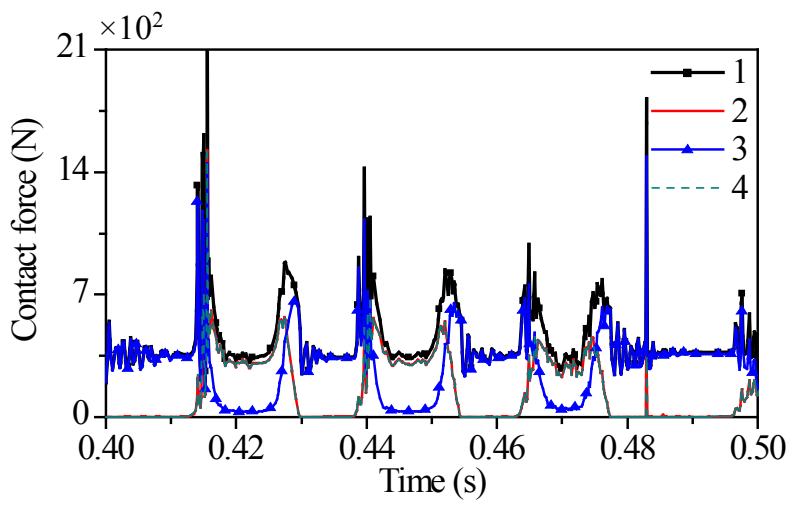

Fig. 5. Contact force of the four clearance joints at the end of numerical simulation

\section{Conclusions}

In this paper, the dynamic characteristics of flap actuation system with four clearance joints are investigated using computational methodology, where the contact force model is described by the "Impactfunction", which consists of a nonlinear normal contact force model and a modified Coulomb friction model. And then the influence of clearances on the system dynamic interaction phenomenon is further analysed and some combination modes are illustrated.

From the numerical simulation results, it can be concluded that the existence of joint clearances will lead to the worse dynamic behaviours of the flap actuation system, namely, angular velocity, angular acceleration and contact force. 1) There exists a strong dynamic interaction between four clearance joints, where the impact mode occurred in one joint will immediately affect other joints. 2) It is clear that the clearance joint nearer to the input component appears higher contact force and four joints present the similar trend of contact force when the system reaches stable, particularly, the contact forces of two joints connected by a rigid rod are almost the same. 3) The system stability can judged by the motion mode of the joint connected with the output component. 4) When one joint is in the continuous contact motion mode, the angular acceleration of this system will coincide with that in the occasion of the joint is considered as ideal.

\section{Acknowledgments}

This research was supported by the National Natural Science Foundation of China (Grant No. 51675429), Key Project of National Natural Science Foundation of China (Grant No. 51535009), the Fundamental Research Funds for the Central Universities (Grant No.31020190504003) and the 111 Project (Grant No. B13044). The authors would like to express their appreciation to the agencies.

\section{References}

1. D. Arriola, F. Thielecke, Model-based design and experimental verification of a monitoring concept for an active-active electromechanical aileron actuation system. Mech Syst Signal Pr, 94, 322-345 (2017)

2. Y. Y. Li, Z. L. Wang, C. Wang, W H Huang, Planar rigid-flexible coupling spacecraft modeling and control considering solar array deployment and joint clearance. Acta Astronaut, 142, 138-151 (2018)

3. Z. F. Bai, J. J. Zhao, J. Chen, Y. Zhao, Design optimization of dual-axis driving mechanism for satellite antenna with two planar revolute clearance joints, Acta Astronaut, 144, 80-89 (2018)

4. B. Li, S. M. Wang, V. Makis, X. Z. Xue, Dynamic characteristics of planar linear array deployable structure based on scissor-like element with differently located revolute clearance joints, Proc IMechE Part C: J Mechanical Engineering Science, 232 (10), 1759-1777 (2018)

5. O. Muvengei, J. Kihiu, B. Ikua, Dynamic analysis of planar rigid-body mechanical systems with twoclearance revolute joints. Nonlinear Dynam, 73, 259-273 (2013)

6. J. Ma, L. F. Qian, Modeling and simulation of planar multibody systems considering multiple revolute clearance joints. Nonlinear Dynam, 90, 1907-1940 (2017)

7. C. Birleanu, S. Talu, Machine elements. Designing and computer assisted graphical representations, Cluj-Napoca, Romania, Victor Melenti Publishing house, 2001. ISBN 973-99539-6-4.

8. T. Nitulescu, S. Talu, Applications of descriptive geometry and computer aided design in engineering graphics, Cluj-Napoca, Romania, Risoprint Publishing house, 2001. ISBN 973-656-102-X.

9. I. Khemili, L. Romdhane, Dynamic analysis of a flexible slider-crank mechanism with clearance. Eur $J$ Mech A-Solid, 27, 882-898 (2008)

10. Y. Chen, Y. Sun, C. Chen, Dynamic analysis of a planar slider-crank mechanism with clearance for a high speed and heavy load press system. Mech Mach Theory, 98, 81-100 (2016)

11. MDI. Building models in ADAMS/View, 2012, ADAMS/Solver online help. 